OPEN ACCESS

Edited by:

Jennifer S. Yokoyama University of San Francisco,

United States

Reviewed by:

Yang Lü,

First Affiliated Hospital of Chongqing

Medical University, China

Byoungjin Park,

Yonsei University Health System,

South Korea

*Correspondence:

Huihui Bao

huihui_bao77@126.com

Xiaoshu Cheng

xiaoshumenfan126@163.com

${ }^{\dagger}$ These authors have contributed equally to this work and share first

authorship

Specialty section:

This article was submitted to Dementia and Neurodegenerative

Diseases,

a section of the journal

Frontiers in Neurology

Received: 29 June 2021 Accepted: 15 December 2021

Published: 03 February 2022

Citation:

Xie Y, Li J, Yu G, Zhou X, Zhou W, Zhu L, Wang T, Huang $X$, Bao $H$ and Cheng X (2022) Association Between

Lipid Accumulation Product and

Cognitive Function in Hypertensive Patients With Normal Weight: Insight From the China H-type Hypertension

Registry Study.

Front. Neurol. 12:732757.

doi: 10.3389/fneur.2021.732757

\section{Association Between Lipid} Accumulation Product and Cognitive Function in Hypertensive Patients With Normal Weight: Insight From the China H-type Hypertension Registry Study

Yanyou Xie ${ }^{1 \dagger}$, Junpei Li ${ }^{1 \dagger}$, Guotao Yu ${ }^{2}$, Xinlei Zhou ${ }^{1}$, Wei Zhou ${ }^{3}$, Lingjuan Zhu ${ }^{3}$, Tao Wang ${ }^{3}$, Xiao Huang ${ }^{1,3}$, Huihui Bao ${ }^{1,3 *}$ and Xiaoshu Cheng ${ }^{1,3 *}$

${ }^{1}$ Department of Cardiovascular, The Second Affiliated Hospital of Nanchang University, Nanchang, China, ${ }^{2}$ Urban Medical Institutions, Jiangwan Public Health Center, Wuyuan, China, ${ }^{3}$ Center for Cardiovascular Disease Prevention and Treatment, The Second Affiliated Hospital of Nanchang University, Nanchang, China

Background: Hypertension is a major cardiovascular risk factor for cognitive impairment. Lipid accumulation product (LAP), an index that represents fat overaccumulation in the body, has been shown to be associated with cardiovascular disease. Nevertheless, the relationship between LAP and cognitive function in hypertensive patients with normal weight has been infrequently studied.

Objective: This study aimed to assess the relationship between LAP and cognitive function in hypertensive patients with normal weight.

Methods: This study included 5,542 Chinese hypertensive patients with normal weight. Cognitive function was evaluated using the Mini-Mental State Examination (MMSE). The relationship between LAP and MMSE scores was evaluated using multiple linear regression.

Results: The mean age of the participants was $64.8 \pm 9.3$ years, and 2,700 were men (48.7\%). The mean MMSE score was $24.5 \pm 5.1$ in men and $19.2 \pm 6.5$ in women. The mean LAP was $26.2 \pm 25.5$ in men and $42.5 \pm 34$ in women. Log $_{10}$-LAP showed a significant positive association with MMSE score (men: $\beta=0.69,95 \% \mathrm{Cl} 0.14-1.24$, $p=0.015$; women: $\beta=1.03,95 \% \mathrm{Cl} 0.16-1.90, p=0.020)$. When LAP was divided into 3 groups according to tertiles, participants in the third LAP tertile had higher MMSE scores for both men ( $p$ for trend $=0.04$ ) and women ( $p$ for trend $=0.015$ ).

Conclusion: LAP showed an independent positive association with MMSE in Chinese hypertensive patients with normal weight.

Keywords: lipid accumulation product, cognitive function, mini-mental state examination, hypertension, normal-weight 


\section{INTRODUCTION}

According to the World Alzheimer Report 2015, 46.8 million people are living with dementia worldwide, and this number is predicted to exceed 131.5 million by 2050 (1). A largesample, multiregional study was conducted in 2019, and it showed that the prevalence of dementia was $5.60 \%$ (3.507.60) for individuals aged $\geq 65$ years in China (2). Dementia is a notable burden for families and society, and substantial evidence indicates that hypertension is a crucial risk factor for dementia. Several cohort studies have suggested that high blood pressure in middle-aged individuals is linked to an increased risk of cognitive decline over time (35).

Obesity is considered a risk factor for dementia $(6,7)$. However, the association between obesity and the risk of dementia is unclear. In late life, there are less apparent adverse effects of obesity, and potential protective effects are observed. A recent meta-analysis reported a positive association between obesity in middle age and subsequent dementia, but a negative association was noted between these two parameters in old age (8). The limitations of traditional anthropometric measurements used to evaluate obesity are obvious. Obesity is usually distinguished by the excessive accumulation of fatty tissue. Body mass index (BMI) cannot be used to differentiate between adipose and lean tissue, and although waist circumference (WC) is a fair indicator of abdominal adipose tissue, it is still unable to correctly reflect whole-body adipose tissue (9, 10). Although imaging examinations are the "gold standard" for the assessment of adipose tissue, they are too costly for extensive use. Lipid accumulation product (LAP) is an index, that is calculated from WC and triglyceride (TG) levels, and it has been proposed as a simple and economical method to evaluate lipid accumulation (11). Studies have indicated that LAP is highly correlated with metabolic syndromes (12), type 2 diabetes mellitus (13), stroke (14), and arterial stiffness (15). Thus, researchers have paid increasing attention to the clinical significance of LAP.

Obesity often occurs along with metabolic abnormality, however, some persons with normal weight may have substantial metabolic disorders that are similar to that of obese people. These individuals are known as metabolically obese normalweight (MONW). Epidemiologic studies have reported that MONW individuals account for $\sim 20 \%$ of the normal-BMI population $(16,17)$. A recent study demonstrated that LAP is an effective marker for identifying the MONW phenotype in Chinese adults (17). Obesity is often associated with hypertension, as either a causative factor or a concomitant disease. Elevated blood pressure has a direct effect on cognitive performance, several relevant studies have reported that hypertension in midlife increased the risk of cognitive dysfunction over time (3-5). However, it remains unknown whether metabolic abnormality in normal-weight individuals may have an impact on cognition function in the hypertensive population. Consequently, this study sought to assess the relationship between LAP and cognitive function in hypertensive participants with a normal weight.

\section{METHODS}

\section{Participants}

The data of this study were derived from the China $\mathrm{H}$ type Hypertension Registry Study (registration number: ChiCTR1800017274). Briefly, the study was a large-scale, realworld observational registry study that aimed to create a national registry of patients with $\mathrm{H}$-type hypertension, investigate the prevalence and control of H-type hypertension in China, and explore related factors that would affect disease prognosis. Details of the methodology of this study had been published elsewhere (18). The enrolled participants fulfilled the following criteria: (1) age $\geq 18$ years and (2) hypertension defined as systolic blood pressure $(\mathrm{SBP}) \geq 140 \mathrm{~mm} \mathrm{Hg}$ and/or diastolic blood pressure (DBP) $\geq 90 \mathrm{~mm} \mathrm{Hg}$, self-reported diagnosis of hypertension previously, or receiving antihypertensive medication at baseline. Participants were excluded based on the following conditions: (1) inability to provide informed consent because of psychological or nervous system impairment and (2) follow-up was notably difficult to complete according to the study protocol. The study was approved by the Ethics Committee of the Biomedical Institute of Anhui Medical University. Written informed consent was obtained from all the study participants.

A total of 10,255 hypertensive patients completed the MiniMental State Examination (MMSE) questionnaire. We excluded participants with missing LAP $(n=3)$ and BMI $(n=2)$ data, and those with stroke $(n=761)$, lipid-lowering drugs $(n=$ $258)$, BMI $\geq 25 \mathrm{~kg} / \mathrm{m}^{2}(n=3,081)$, or BMI $<18.5 \mathrm{~kg} / \mathrm{m}^{2}(n$ $=624$ ). Ultimately, 5,542 subjects were included in the analysis (Figure 1).

\section{Data Collection}

The research staff used questionnaires to gather information, namely, demographic characteristics, lifestyle data, medication history, medical history, and MMSE. The anthropometric indices, namely, height, weight, WC, SBP, and DBP, were measured by trained investigators. Blood pressure was obtained by electronic sphygmomanometers on the right arm placed at the heart level in the sitting position after the participants rested for at least $5 \mathrm{~min}$; the blood pressure was calculated as the mean of three consecutive measurements.

After an overnight fast of at least $8 \mathrm{~h}$, venous blood samples were collected from all study participants by trained research staff and sent to the Biaojia Biotechnology Laboratory, which is located in Shenzhen, China. Lipid profiles [total cholesterol, TG, low-density lipoprotein-cholesterol (LDL-C), and highdensity lipoprotein-cholesterol (HDL-C)], fasting blood glucose, homocysteine, and creatinine levels were measured using an automatic clinical assay.

Lipid accumulation product (LAP) was calculated based on fasting TG and WC values using the following formula: $\mathrm{LAP}=$ [WC $(\mathrm{cm})-65] \times \mathrm{TG}(\mathrm{mmol} / \mathrm{l})$ for men and [WC $(\mathrm{cm})-58$ ] $\times \mathrm{TG}(\mathrm{mmol} / \mathrm{l})$ for women (11). A total of $104 \mathrm{men}$ had WC $\leq$ $65 \mathrm{~cm}$ and 16 women had WC $\leq 58 \mathrm{~cm}$. WC values for men that were $\leq 65 \mathrm{~cm}$ were reassigned to $66 \mathrm{~cm}$ and those WC values for women that were $\leq 58 \mathrm{~cm}$ were reassigned to $59 \mathrm{~cm}$ to generate a LAP-value $>0$ and enable the logarithmic transformation of 


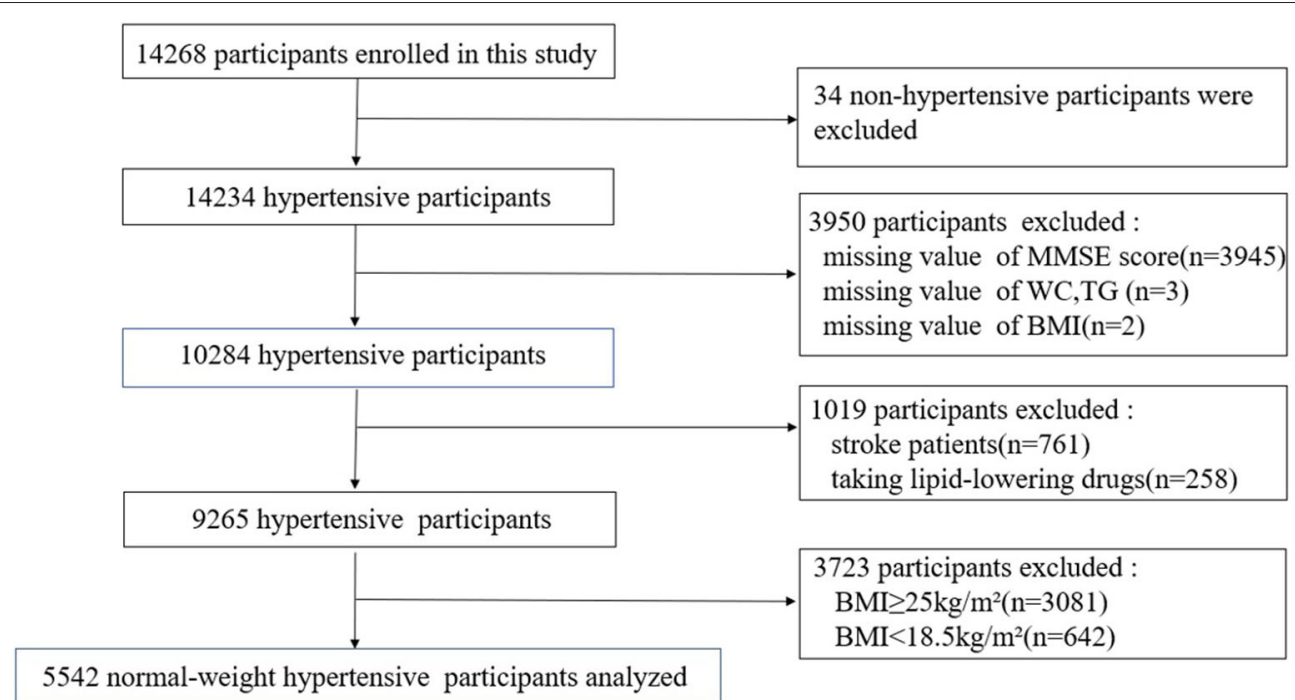

FIGURE 1 | Flow chart of the study participants.

LAP. Diabetes was defined as either fasting blood glucose $\geq 7.0$ $\mathrm{mmol} / \mathrm{l}$, self-report of a physician diagnosis, or self-report use of antidiabetic medication.

\section{Assessment of Cognitive Function}

Cognitive function was assessed by the Chinese MMSE version in this study. This tool has been widely used because of its proven high reliability and has been validated for use in the Chinese population (19). MMSE consisted of the following cognitive domains: orientation (10 points), immediate recall (3 points), short-term verbal memory ( 3 points), visuospatial memory (1 point), language ( 8 points), and attention and calculation (5 points). MMSE scores ranged from 0 to 30 points, with higher scores indicating better cognition.

\section{Covariate}

The selected covariates included age, sex, education (primary school graduate or below, middle/high/special school, and college graduate or above), current drinking (yes or no), current smoking (yes or no), SBP, DBP, diabetes (yes or no), coronary heart disease (yes or no), antihypertensive drug (yes or no), homocysteine, total cholesterol, HDL-C, LDL-C, and estimated glomerular filtration rate (eGFR).

\section{Statistical Analysis}

The baseline characteristics of the total population are presented as mean \pm SD for continuous variables and as percentages (\%) for categorical variables. ANOVA or the chi-squared test was used to examine the differences between the groups by LAP tertiles. Because LAP exhibited a skewed distribution, logarithmic data were used for the statistical analyses. Because LAP and the MMSE score have significant discrepancies with respect to sex, all subsequent analyses were performed separately for men and women. Multivariate linear regression models were used to evaluate the association of LAP with the MMSE score, and the results are presented as $\beta$ and $95 \%$ CIs. All the analyses consisted of three statistical models. The crude model was not adjusted, model 1 was adjusted for age and education, and model 2 was adjusted for age, education, drinking status, smoking status, SBP, DBP, CHD, diabetes, an antihypertensive drug, homocysteine, total cholesterol, LDL-C, HDL-C, and eGFR. Trend tests were conducted using LAP tertile categories as continuous data. The dose-response association of LAP and the MMSE score was performed using a fitted smoothing curve. Stratified analyses were performed in various subgroups, namely, age, drinking status, smoking status, SBP, DBP, diabetes, total cholesterol, and eGFR.

$\mathrm{R}$ version 3.4.3 (R Foundation for Statistical Computing, Vienna, Austria) and EmpowerStates (www.empowerstats.com) were used to conduct the data analyses. Only $p<0.05$ (two-sided) were deemed as statistically significant.

\section{RESULTS}

\section{Baseline Characteristics}

Of the 5,542 participants, 2,700 (48.7\%) were men and 2,842 (51.3\%) were women, and the mean age of the participants was $64.8 \pm 9.3$ years. The baseline characteristics of the study participants were distributed according to sex and LAP tertiles as presented in Table 1. The LAP tertile ranges were $\leq 13.3,13.3-$ 27.2 , and $\geq 27.2$ in the men group and $\leq 25.1,25.1-45.2$, and $\geq 45.2$ in the women group. In the men group, participants with higher LAP levels had higher BMI, WC, DBP, eGFR, TG, total cholesterol, and MMSE scores, and were more likely to have diabetes and a higher educational level. However, they had lower values for age, SBP, and HDL-C and were less likely to smoke. In the women group, participants with higher LAP levels had higher BMI, WC, DBP, TG, total cholesterol, and MMSE scores, and were more likely to have diabetes. In contrast, participants with higher LAP levels had lower values of age and HDL-C. 
TABLE 1 | Baseline characteristics of study participants.

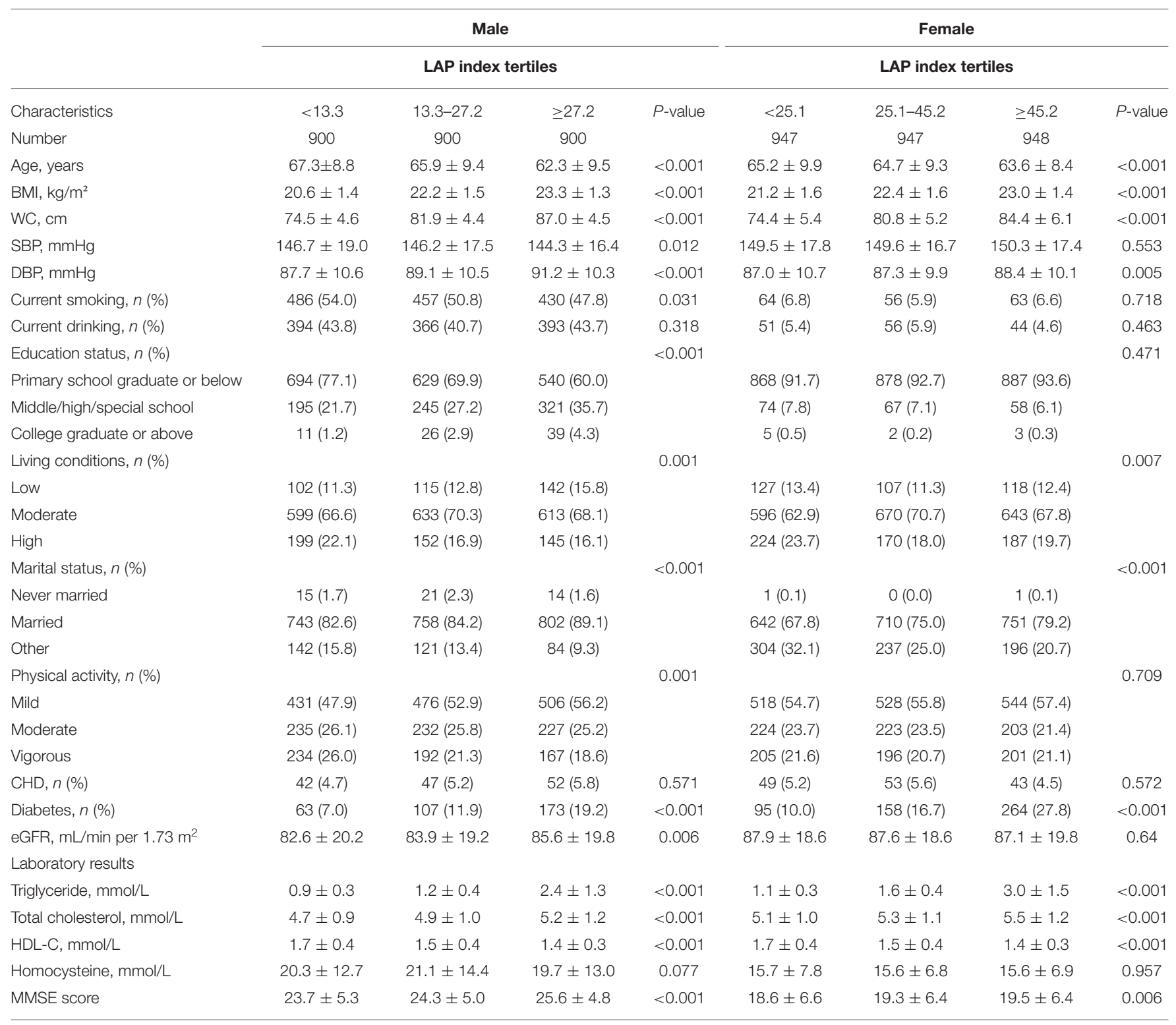

LAP, lipid accumulation product; BMI, body mass index; SBP, systolic blood pressure; DBP, diastolic blood pressure; CHD, coronary heart disease; HDL, high-density lipoprotein cholesterol.

\section{Association Between LAP and MMSE Score}

The results of the linear regression analysis investigating the association of LAP with MMSE score are shown in Table 2. In the men group, $\log _{10}$-LAP $(\beta=2.03,95 \%$ CI $1.55-2.51)$ had a significant positive association with the MMSE score in the crude model. After adjustment for age and education, this association remained significant $(\beta=1.03,95 \%$ CI 0.57 1.48). With additional adjustment for smoking status, drinking status, SBP, DBP, CHD, diabetes, an antihypertensive drug, homocysteine, total cholesterol, HDL-C, LDL-C, and eGFR, this association was still significant $(\beta=0.69,95 \%$ CI $0.14-1.24)$. Likewise, The regression coefficient $(\beta)$ and $95 \% \mathrm{CI}$ of the relationship of $\log _{10}$-LAP with MMSE score was (1.23, $0.45-$ $2.02),(1.11,0.39-1.82)$, and $(1.03,0.16-1.90)$ for the crude model, model 1, and model 2, respectively, in the women group. When LAP was assessed as tertiles (Table 2), LAP was positively associated with MMSE score among the men ( $p$ for trend $=0.04$ ) and women ( $p$ for trend $=0.015$ ). Figure 2 shows a positive association between LAP and MMSE score in both men and women.

\section{Subgroup Analyses}

In the stratified analysis, we further assessed the relationship between LAP and MMSE scores according to various subgroups. 
TABLE 2 | Association between LAP as a common logarithm-transformed continuous variable and cognitive function represented by MMSE scores.

\begin{tabular}{|c|c|c|c|c|c|c|c|c|}
\hline \multirow[t]{2}{*}{ Lg LAP index } & \multirow[t]{2}{*}{$N$} & \multirow[t]{2}{*}{ MMSE scores (Mean \pm SD) } & \multicolumn{2}{|c|}{ Crude model } & \multicolumn{2}{|c|}{ Model 1} & \multicolumn{2}{|c|}{ Model 2} \\
\hline & & & $\beta(95 \% \mathrm{Cl})$ & $P$-value & $\beta(95 \% \mathrm{Cl})$ & $P$-value & $\beta(95 \% \mathrm{Cl})$ & $P$-value \\
\hline \multicolumn{9}{|l|}{ Male } \\
\hline Continuous & 2,700 & $24.5 \pm 5.1$ & $2.03(1.55,2.51)$ & $<0.001$ & $1.03(0.57,1.48)$ & $<0.001$ & $0.69(0.14,1.24)$ & 0.015 \\
\hline \multicolumn{9}{|l|}{ Tertiles } \\
\hline $\mathrm{T} 1$ & 900 & $23.7 \pm 5.3$ & O(ref.) & & $\mathrm{O}$ (ref.) & & O(ref.) & \\
\hline T2 & 900 & $24.3 \pm 5.0$ & $0.62(0.16,1.09)$ & 0.009 & $0.26(-0.17,0.69)$ & 0.233 & $0.08(-0.37,0.52)$ & 0.732 \\
\hline T3 & 900 & $25.6 \pm 4.8$ & $1.88(1.41,2.34)$ & $<0.001$ & $0.95(0.51,1.39)$ & $<0.001$ & $0.57(0.06,1.09)$ & 0.030 \\
\hline$P$ for trend & & & $<0.001$ & & $<0.001$ & & 0.040 & \\
\hline \multicolumn{9}{|l|}{ Female } \\
\hline Continuous & 2,842 & $17.6 \pm 6.4$ & $1.23(0.45,2.02)$ & 0.002 & $1.11(0.39,1.82)$ & 0.002 & $1.03(0.16,1.90)$ & 0.020 \\
\hline \multicolumn{9}{|l|}{ Tertiles } \\
\hline $\mathrm{T} 1$ & 947 & $18.6 \pm 6.6$ & O(ref.) & & O(ref.) & & O(ref.) & \\
\hline $\mathrm{T} 2$ & 947 & $19.3 \pm 6.4$ & $0.71(0.13,1.29)$ & 0.017 & $0.71(0.18,1.24)$ & 0.009 & $0.66(0.11,1.21)$ & 0.020 \\
\hline T3 & 948 & $19.5 \pm 6.4$ & $0.90(0.32,1.48)$ & 0.003 & $0.84(0.31,1.37)$ & 0.002 & $0.77(0.15,1.38)$ & 0.015 \\
\hline$P$ for trend & & & $<0.001$ & & $<0.001$ & & 0.015 & \\
\hline
\end{tabular}

Model 1, adjusted for age, education.

Model 2, adjusted for age, education, smoking status, drinking status, SBP, DBP, CHD, diabetes, anti-hypertensive drug, homocysteine, LDL-C, HDL-C, TCHO, eGFR.

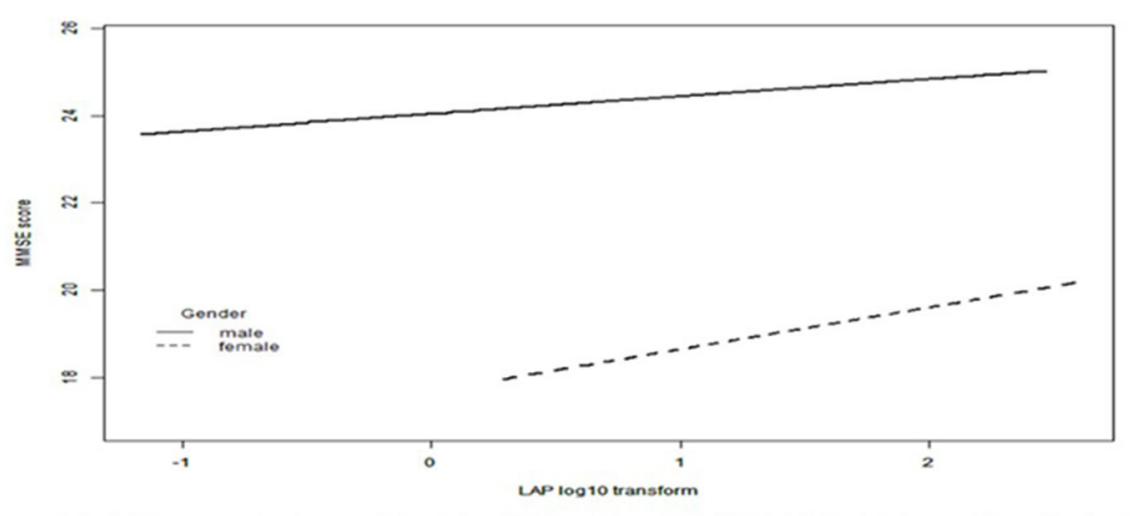

Adjusted for: age, education, smoking status, drinking status, SBP, DBP, CHD, diabetes, anti-hypertensive drug , homocysteine, LDL-C, HDL-C, TCHO, eGFR

FIGURE 2 | Fitted curves for LAP index as a common logarithm-transformed continuous variable and cognitive function represented by MMSE scores. Adjusted for: age, education, smoking status, drinking status, SBP, DBP, CHD, diabetes, anti-hypertensive drug, homocysteine, LDL-C, HDL-C, TCHO, eGFR.

As presented in Tables 3, 4, except for the age subgroup in women $(p$ for interaction $=0.008$ ), significant differences were not found for drinking status, smoking status, diabetes, SBP, DBP, total cholesterol, and eGFR regardless of sex (all $P$ for interaction $>0.05)$. Women $\geq 65$ years showed significantly higher MMSE scores ( $\beta=0.98,95 \%$ CI 0.04-1.92) compared to women $<65$ years.

\section{DISCUSSION}

In this cross-sectional study, we found a significant positive association between LAP and cognitive function in normalweight hypertensive patients. In addition, our results suggested that the positive relationship between LAP and cognitive function was more prominent in women aged $\geq 65$ years.

Several studies have assessed the impact of obesity on cognitive function. A prospective study conducted by Whitmer et al. showed that obese individuals in midlife showed an increased risk of future cognitive impairment among adults in America (20). However, another observational study performed by $\mathrm{Hou}$ et al. demonstrated that being overweight was independently associated with a reduced risk of cognitive impairment in elderly Chinese individuals (21). In addition, a longitudinal study by Luchsinger et al. reported the lack of an association between BMI and late-life dementia in the elderly in America (22). The relationship between adiposity, defined using BMI in the above studies, and cognitive function was analyzed in middle and late life, and the 
TABLE 3 | Stratified analyses of association between LAP as a common logarithm-transformed continuous variable and cognitive function represented by MMSE scores in male.

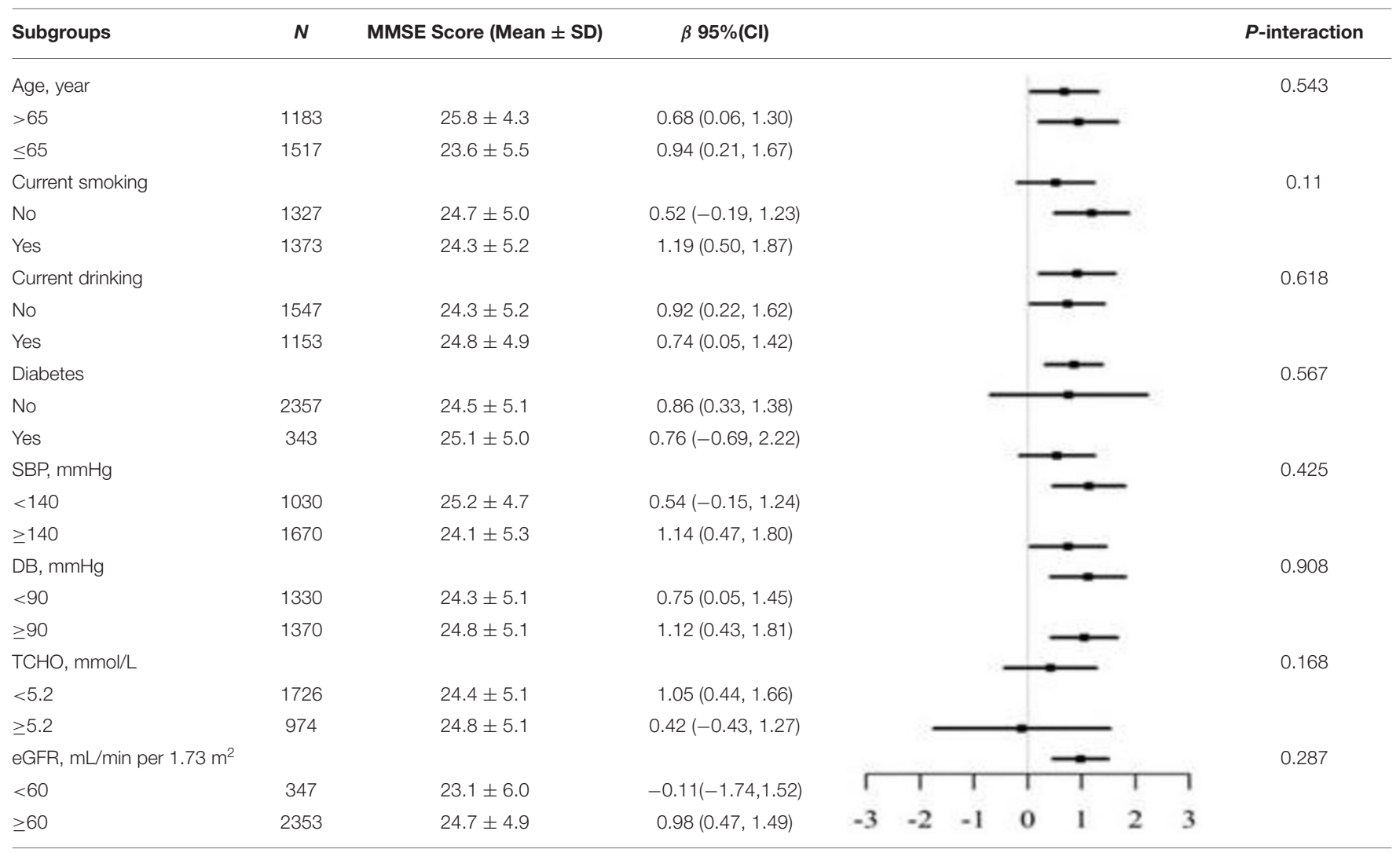

Adjusted for: age, education, smoking status, drinking status, SBP, DBP, CHD, diabetes, anti-hypertensive drug, homocysteine, LDL-C, HDL-C, TCHO, eGFR.

results were conflicting. The discrepancies in these findings might be attributed to variations in countries, races, study sizes, adjusted variables, or participant ages. In addition, the elderly usually have decreased muscle weight and increased fat weight. However, BMI does not reflect body fat accumulation, and it does not differentiate between adipose and lean tissue. LAP represents both the anatomical and physiological changes associated with fat overaccumulation in the body. Compared to BMI, LAP is more appropriate for explaining the relationship between obesity and cognitive function. It is well-known that increased weight might be due to the enlargement of lean tissues or systemic overload of fluid. A longitudinal Icelandic study also showed that a higher amount of muscle was associated with a lower risk of dementia in men and women (23). This may explain why the relationship between BMI and cognitive function was inconsistent in previous studies.

Therefore, it is important to explore the relationship between LAP and cognitive function among normal-weight individuals. However, data on the role of LAP in cognitive function among hypertensive populations are scarce, and our study is the first to explore the association between LAP and cognitive function in hypertensive patients. Our results were in line with a prospective study conducted in 16,791 hypertensive participants in China, which showed that obesity defined by BMI was independently related to slower cognitive decline in Chinese adult patients with hypertension (24). Furthermore, Yu et al. (25) conducted a crosssectional study to examine the relationship between LAP and mild cognitive impairment in 220 patients with type 2 diabetes and found that higher LAP was related to an increased risk of mild cognitive impairment in patients with type 2 diabetes. The inconsistent results might be due to the differences in the study population, and the sample size was relatively small; thus, large sample size may yield clearer results. While the sample size was 5,542 in this study, our result that higher LAP was related to better cognitive function in hypertensive participants seems plausible.

A possible mechanism underlying the relationship between LAP and cognitive function may be linked to leptin, which is produced by adipose tissue. Some studies have indicated that leptin could promote synaptogenesis and neurogenesis in the hippocampus, which is related to learning and memory, thus facilitating cognitive function (26). Tau hyperphosphorylation and $\beta$-amyloid peptide deposition have been implicated in the pathogenesis of Alzheimer's disease (27). Several laboratory studies have indicated that leptin reduces tau phosphorylation and $\beta$-amyloid peptide production. These attributes of leptin in the brain confer protection against the risk of Alzheimer's disease 
TABLE 4 | Stratified analyses of association between LAP as a common logarithm-transformed continuous variable and cognitive function represented by MMSE scores in female.

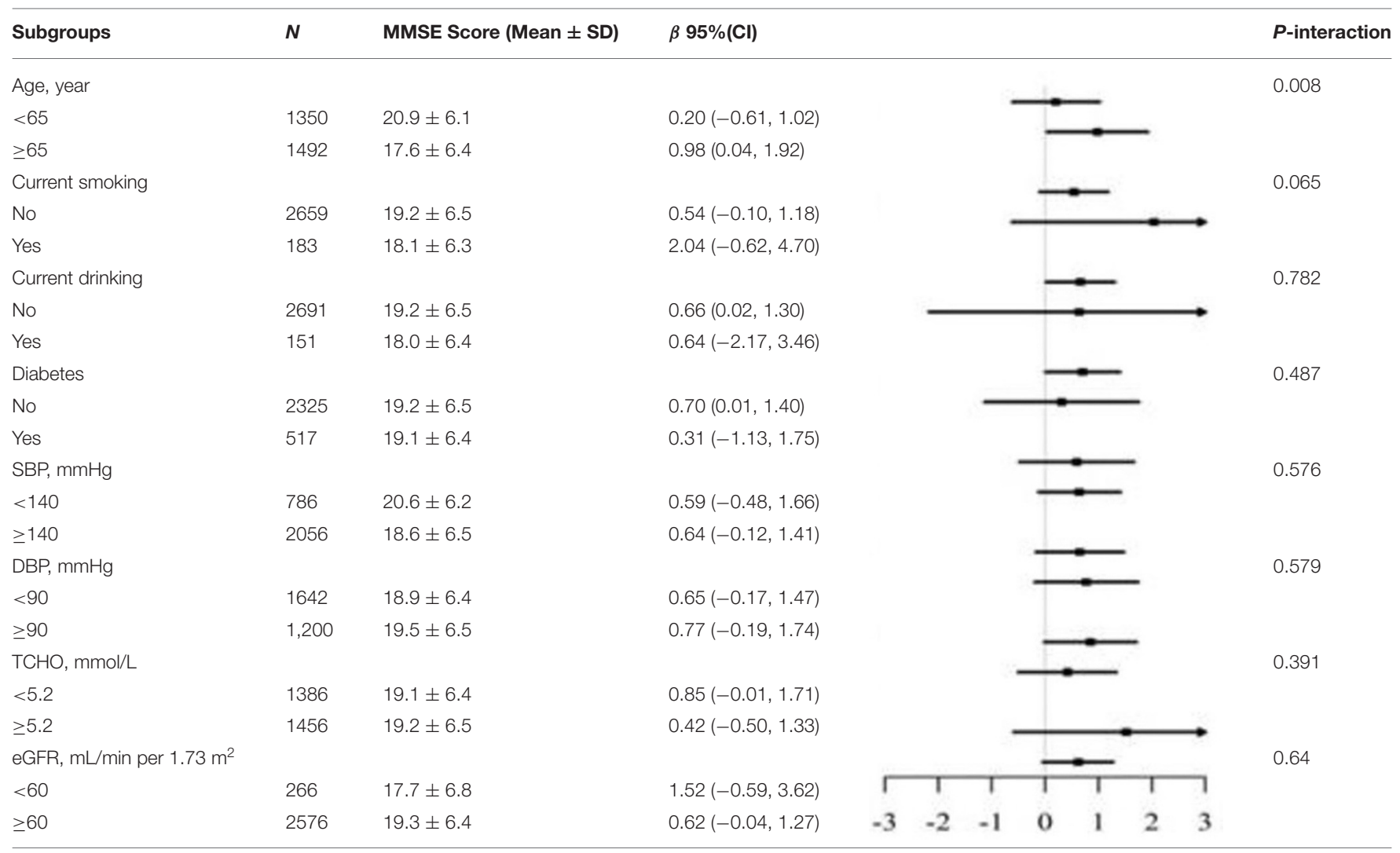

Adjusted for: age, education, smoking status, drinking status, SBP, DBP, CHD, diabetes, anti-hypertensive drug, homocysteine, LDL-C, HDL-C, TCHO, eGFR.

(26). Another possible explanation is that the accumulation of low adipose tissue in the body may be attributed to preclinical dementia and other preexisting illnesses (28). This could directly or indirectly disrupt the energy balance through neuronal dysfunction and neurodegeneration. These include disturbances in cognition, motion, psychology, and pathology of the energy balance center in the hypothalamus. People may forget to eat or lack the desire to eat because of the loss of olfaction and gustation.

In our study, older women (aged $\geq 65$ years) had larger regression coefficients for LAP in relation to MMSE scores than younger women. The sex difference in estrogen is a possible explanation for this phenomenon. Adipose tissue is the major source of estrogen in postmenopausal women $(29,30)$. Estrogen has been demonstrated to play an important role in the bioenergetic system of the brain, namely, glucose transport, glycolysis, the tricarboxylic citric acid cycle, oxidative phosphorylation, and ATP production (31). In addition, body fat loss preceding the onset of the clinical syndrome of dementia by as much as 10 years may also be related to this phenomenon (32). Therefore, the higher LAP in older women with normal weight was associated with better cognitive function than in younger women.
This study has several limitations. First, the sensitivity and specificity of the MMSE for identifying mild cognitive impairment may be limited. Our study participants mainly came from the rural areas in China, where people were more likely to be less well-educated. While the MMSE is proved to be a useful tool for assessing cognitive function in the low educated population (33), so it may be more appropriate for the population of this study. Further research combining MMSE and other cognitive function rating scales, such as the Montreal Cognitive Assessment or Mini-Cog, is needed in the future to validate the result. Second, this was a cross-sectional study, and it failed to identify any causal relationship between LAP and cognitive function in normalweight hypertensive individuals. Third, although we carefully adjusted for several important confounders using various statistical models, there may still be potential confounding factors that were not considered. Fourth, lack of information on the type of glucose-lowering drugs for diabetics. Thus, it was unable to evaluate the impact of specific glucose-lowering drugs in this study. Finally, this study was conducted in hypertensive populations, our findings may not be appropriate to generalize to other populations. However, hypertension is a significant risk factor for dementia, and more attention needs be paid to this population. 


\section{CONCLUSION}

Overall, LAP demonstrated a significant positive relationship with cognitive function in normal-weight hypertensive persons. The results indicate that appropriate lipid accumulation in normal-weight individuals is associated with better cognitive function. The results obtained from this study may be valuable for the prevention of cognitive dysfunction.

\section{DATA AVAILABILITY STATEMENT}

The raw data supporting the conclusions of this article will be made available by the authors, without undue reservation.

\section{ETHICS STATEMENT}

The studies involving human participants were reviewed and approved by Ethics Committee of the Biomedical Institute of Anhui Medical University. The patients/participants provided their written informed consent to participate in this study.

\section{AUTHOR CONTRIBUTIONS}

$\mathrm{XC}$ and $\mathrm{HB}$ contributed to the design of the study and reviewed the article. YX and JL were responsible for the

\section{REFERENCES}

1. Prince M, Wimo A, Guerchet M, Ali G, Wu Y, Prina M, et al. World Alzheimer Report 2015. The Global Impact of Dementia. An Analysis of Prevalence, Incidence, Cost \& Trends. London: Alzheimer's Disease International (ADI) (2015).

2. Huang Y, Wang Y, Wang H, Liu Z, Yu X, Yan J, et al. Prevalence of mental disorders in China: a cross-sectional epidemiological study. Lancet Psychiat. (2019) 6:211-24. doi: 10.1016/S2215-0366(18)30511-X

3. Knopman DS, Mosley TH, Catellier DJ, Coker LH. Fourteen-year longitudinal study of vascular risk factors, APOE genotype, and cognition: the ARIC MRI Study. Alzheimer's Dement. (2009) 5:207-14. doi: 10.1016/j.jalz.2009. 01.027

4. Swan GE, Decarli C, Miller BL, Reed T, Wolf PA, Jack LM, et al. Association of midlife blood pressure to late-life cognitive decline and brain morphology. Neurology. (1998) 51:986-93. doi: 10.1212/WNL.51.4.986

5. Gottesman RF, Schneider ALC, Albert M, Alonso A, Bandeen-Roche K, Coker L, et al. Midlife hypertension and 20-Year cognitive change: the atherosclerosis risk in communities neurocognitive study. JAMA Neurol. (2014) 71:1218. doi: 10.1001/jamaneurol.2014.1646

6. Isaac V, Sim S, Zheng H, Zagorodnov V, Tai ES, Chee $M$, et al. Adverse associations between visceral adiposity, brain structure, and cognitive performance in healthy elderly. Front Aging Neurosci. (2011) 3:12. doi: 10.3389/fnagi.2011.00012

7. Yoon DH, Choi SH Yu JH, Ha JH Ryu SH, Park DH, et al. The relationship between visceral adiposity and cognitive performance in older adults. Age Ageing. (2012) 41:456-61. doi: 10.1093/ageing/afs018

8. Nishizawa A, Cuelho A, Farias-Itao DS, Campos FM, Leite REP, FerrettiRebustini REL, et al. Direct measurements of abdominal visceral fat and cognitive impairment in late life: findings from an autopsy study. Front Aging Neurosci. (2019) 11:109. doi: 10.3389/fnagi.2019. 00109 data analysis and edited this article. XZ contributed to the article and data review. GY contributed to the data collection. LZ and TW contributed to the data interpretation. $\mathrm{XH}$ and WZ revised the article for scientific and logical accuracy. All authors read and approved the final version of the manuscript.

\section{FUNDING}

This study was supported by the National Natural Science Foundation of China (81760049), Jiangxi Science and Technology Innovation Platform Project (20165BCD41005), National Key R\&D Program of China (2018YFC1312902), and Key Project of the Education Department of Jiangxi Province (GJJ170013).

\section{ACKNOWLEDGMENTS}

The authors are grateful to all the participants for their cooperation in this study.

\section{SUPPLEMENTARY MATERIAL}

The Supplementary Material for this article can be found online at: https://www.frontiersin.org/articles/10.3389/fneur. 2021.732757/full\#supplementary-material

9. Xia C, Li R, Zhang S, Gong L, Ren W, Wang Z, et al. Lipid accumulation product is a powerful index for recognizing insulin resistance in non-diabetic individuals. Eur J Clin Nutr. (2012) 66:1035-38. doi: 10.1038/ejcn.2012.83

10. Nusrianto R, Ayundini G, Kristanti M, Astrella C, Amalina N, MuhadiSoewondo P, et al. Visceral adiposity index and lipid accumulation product as a predictor of type 2 diabetes mellitus: the Bogor cohort study of non-communicable diseases risk factors. Diabetes Res Clin Pr. (2019) 155:1077-98. doi: 10.1016/j.diabres.2019.107798

11. Kahn HS. The "lipid accumulation product" performs better than the body mass index for recognizing cardiovascular risk: a population-based comparison. BMC Cardiovasc Disord. (2005) 5:26. doi: 10.1186/1471-2261-5-26

12. Ray L, Ravichandran K, Nanda SK. Comparison of lipid accumulation product index with body mass index and waist circumference as a predictor of metabolic syndrome in indian population. Metab Syndr Relat Disord. (2018) 16:240-45. doi: 10.1089/met.2017.0119

13. Lee JW, Lim NK, Park HY. The product of fasting plasma glucose and triglycerides improves risk prediction of type 2 diabetes in middle-aged Koreans. Bmc Endocr Disord. (2018) 18:33. doi: 10.1186/s12902-018-0259-X

14. Zhong C, Xia W, Zhong X, Xu T, Li H, Zhang M, et al. Lipid accumulation product and hypertension related to stroke: A 92-Year prospective study among mongolians in China. J Atheroscler Thromb. (2016) 23:830-38. doi: $10.5551 /$ jat.33514

15. Cicero AF, D’Addato S, Reggi A, Marchesini G, Borghi C. Gender difference in hepatic steatosis index and lipid accumulation product ability to predict incident metabolic syndrome in the historical cohort of the Brisighella Heart Study. Metab Syndr Relat Disord. (2013) 11:412-16. doi: 10.1089/met.2012.0147

16. Zheng Q, Lin W, Liu C, Zhou Y, Chen T, Zhang L, et al. Prevalence and epidemiological determinants of metabolically obese but normalweight in Chinese population. BMC Public Health. (2020) 20:487. doi: 10.1186/s12889-020-08630-8 
17. Du T, Yu X, Zhang J, Sun X. Lipid accumulation product and visceral adiposity index are effective markers for identifying the metabolically obese normal-weight phenotype. Acta Diabetol. (2015) 52:855-63. doi: 10.1007/s00592-015-0715-2

18. Li J, Yu S, Zhou W, Zhu L, Wang T, Bao H, et al. U-Shaped association of body mass index with the risk of peripheral arterial disease in Chinese hypertensive population. Int J Gen Med. (2021) 14:3627-34. doi: 10.2147/IJGM. S323769

19. Katzman R, Zhang MY. Ouang-Ya-Qu, Wang ZY, Liu WT, Yu E, et al. A Chinese version of the Mini-Mental State Examination; Impact of illiteracy in a Shanghai dementia survey. J Clin Epidemiol. (1988) 41:971. doi: 10.1016/0895-4356(88)90034-0

20. Whitmer RA, Gunderson EP, Barrett-Connor E, Quesenberry CP, Yaffe K. Obesity in middle age and future risk of dementia: a 27 year longitudinal population based study. BMJ. (2005) 330:1360. doi: 10.1136/bmj.38446.466238.E0

21. Hou Q, Guan Y, Yu W, Liu X, Wu L, Xiao M, et al. Associations between obesity and cognitive impairment in the Chinese elderly: an observational study. Volume. (2019) 14:367-73. doi: 10.2147/CIA.S192050

22. Luchsinger JA, Patel B, Tang MX, Schupf N, Mayeux R. Measures of adiposity and dementia risk in elderly persons. Arch Neurol. (2007) 64:392-98. doi: 10.1001/archneur.64.3.392

23. Spauwen PJ, Murphy RA, Jónsson PV, Sigurdsson S, Garcia ME, Eiriksdottir GL, et al. Associations of fat and muscle tissue with cognitive status in older adults: the AGES-Reykjavik study. Age Ageing. (2017) 46:250-57. doi: 10.1093/ageing/afw219

24. Zhang J, Tang G, Xie H, Wang B, He M, Fu J, et al. Higher adiposity is associated with slower cognitive decline in hypertensive patients: secondary analysis of the china stroke primary prevention trial. J Am Heart Assoc. (2017) 6:5561. doi: 10.1161/JAHA.117.005561

25. Yu Z, Li X, Wang Y, Fu Y, Gao X. Association between lipid accumulation product and mild cognitive impairment in patients with type 2 diabetes. $J$ Alzheimers Dis. (2020) 77:367-74. doi: 10.3233/JAD-200332

26. Marwarha G, Ghribi O. Leptin signaling and Alzheimer's disease. Am J Neurodegener Dis. (2012) 1:245-65. Available online at: https://www.ncbi.nlm. nih.gov/pmc/articles/PMC3560472/

27. Kang S, Lee YH, Lee JE. Metabolism-Centric overview of the pathogenesis of alzheimer's disease. Yonsei Med J. (2017) 58:479-88. doi: $10.3349 /$ ymj.2017.58.3.479
28. Johnson DK, Wilkins $\mathrm{CH}$, Morris JC. Accelerated weight loss may precede diagnosis in Alzheimer disease. Arch Neurol. (2006) 63:1312-17. doi: 10.1001/archneur.63.9.1312

29. Newton CJ, Samuel DL, James VH. Aromatase activity and concentrations of cortisol, progesterone and testosterone in breast and abdominal adipose tissue. J Steroid Biochem. (1986) 24:1033-39. doi: 10.1016/0022-4731(86)90356-0

30. Szymczak J, Milewicz A, Thijssen JH, Blankenstein MA, Daroszewski J. Concentration of sex steroids in adipose tissue after menopause. Steroids. (1998) 63:319-21. doi: 10.1016/s0039-128x(98)00019-1

31. Brinton RD. The healthy cell bias of estrogen action: mitochondrial bioenergetics and neurological implications. Trends Neurosci. (2008) 31:52937. doi: 10.1016/j.tins.2008.07.003

32. Knopman DS, Edland SD, Cha RH. Petersen RC, Rocca WA. Incident dementia in women is preceded by weight loss by at least a decade. Neurology. (2007) 69:739-46. doi: 10.1212/01.wnl.0000267661.65586.33

33. Kim J, Sunwoo M, Sohn Y, Lee P, Hong J. The MMSE and MoCA for screening cognitive impairment in less educated patients with Parkinson's disease. J Mov Disord. (2016) 9:152-9. doi: 10.14802/jmd. 16020

Conflict of Interest: The authors declare that the research was conducted in the absence of any commercial or financial relationships that could be construed as a potential conflict of interest.

Publisher's Note: All claims expressed in this article are solely those of the authors and do not necessarily represent those of their affiliated organizations, or those of the publisher, the editors and the reviewers. Any product that may be evaluated in this article, or claim that may be made by its manufacturer, is not guaranteed or endorsed by the publisher.

Copyright (c) $2022 \mathrm{Xie}, \mathrm{Li}, \mathrm{Yu}$, Zhou, Zhou, Zhu, Wang, Huang, Bao and Cheng. This is an open-access article distributed under the terms of the Creative Commons Attribution License (CC BY). The use, distribution or reproduction in other forums is permitted, provided the original author(s) and the copyright owner(s) are credited and that the original publication in this journal is cited, in accordance with accepted academic practice. No use, distribution or reproduction is permitted which does not comply with these terms. 